\title{
Continuous and fixed ratio reinforcement effects in extinction one day and three weeks after acquisition'
}

EDWIN G. AIKEN AND KIRK L. GIBSON

SAN DIEGO STATE COLLEGE

\begin{abstract}
The influence on response rate during extinction of the reinforcement schedule during acquisition and the time interval between acquisition and extinction was studied in a 2 by 2 factorial design with each condition represented by 10 female albino rats. Two groups were continuously reinforced and 2 groups were intermittently reinforced on a graduated schedule terminating in a fixed ratio-5 schedule at the end of acquisition. Under each reinforcement contingency, 1 subgroup was extinguished $24 \mathrm{hr}$. after acquisition and another 3 weeks after acquisition. An analysis of variance on the rate of responding in the first $35 \mathrm{~min}$. of extinction revealed significant influences attributable to reinforcement schedule and to the interaction of schedule with time interval; the latter arising from a greater response rate with increased acquisition to extinction time interval following the continuous reinforcement contingency and a lesser rate following the intermittent contingency. The results are interpreted in terms of changes over time in the stimulus generalization gradients of the stimuli controlling the behavior chains associated with the 2 reinforcement schedules.
\end{abstract}

Recently, Gleitman and his associates (Gleitman \& Bernheim, 1963; Gleitman, Steinman, \& Bernheim, 1965) have demonstrated that the typical rate accelerations of a bar-press response under a fixed interval reinforcement contingency decline 24 days after the acquisition of the response. The decline in rate scalloping was due to an increase in immediate post-reinforcement responding, so that in terms of overall response rate, an increase was manifested after the delay interval. The research reported here examines the retention of fixed ratio reinforcement effects during extinction, employing a continuously reinforced group for comparison.

\section{Method}

Subjects

Ss were 40 female albino rats of the Holtzman strain. They ranged from 80 to 180 days of age at the start of deprivation. They were weighed three times weekly and maintained at $85 \%$ of their free feeding body weight for the duration of the experiment.

Apparatus

A Davis operant conditioning chamber Mod. DB-103 with associated PD-109A pellet dispenser was covered by a sound deadening box with a top hatch for placing the animal in the chamber and a side door for observation during magazine training. A stopwatch and Hunter
Mod. 141 electrical counter were used to record time and number of responses. A white noise generator was used to mask the sounds of E's movements and part of the counter noise.

Design and Procedure

Two variables-reinforcement schedule and the time between acquisition and extinction-were combined in a 2 by 2 factorial design. Ss were run in 10 sets of four randomly selected animals, with each condition of the design represented in each set. Two Ss in each set were continuously reinforced (CRF) and two intermittently reinforced on a graduating schedule which terminated in a fixed ratio-5 (FR-5) schedule at the end of acquisition. One of the two animals on each of the reinforcement schedules was extinguished $24 \mathrm{hr}$. after acquisition ( 1 day delay), and the other three weeks after acquisition (21 day delay). To equate time on deprivation at extinction, the 1 day delay Ss were brought to $85 \%$ satiated body weight four weeks prior to training, while the 21 day delay Ss were placed on deprivation one week prior to training. For four days after extinction, Ss were allowed free access to food and then weighed as a check on their deprivation state just prior to extinction.

Three days prior to training, all Ss received five 45 $\mathrm{mg}$ Noyes pellets in their home cages before their regular ration. On day 1 all Ss were magazine trained with 50 Noyes pellets. Throughout the experiment, each $\mathrm{S}$ was carried in its home cage to the experimental room and returned immediately after its training session.

Number of reinforcements was equated during acquisition. On days 2,3 , and 4 all Ss received 50 reinforcements. On day 2 all Ss were on a CRF contingency. On day 3 , two Ss in each set were shifted to an FR-3 schedule, and on day 4 to an FR-5 schedule, while the remaining two Ss continued on the CRF contingency. On day 5 the 1 day delay Ss were extinguished to a criterion of $10 \mathrm{~min}$. without responding, and on day 25 the 21 day delay Ss received the same treatment.

The clicking sound of the pellet dispenser was absent on non-reinforced responses, although the relay mechanism of the dispenser plus the counter still produced a clicking sound somewhat softer than that of the dispenser, relay, and counter combined.

\section{Results}

Acquisition

An analysis of variance on rate of response on the first day of acquisition (when all groups were under a CRF contingency) revealed no reliable differences be- 
tween groups. The same analysis on the first 50 responses on the final day of acquisition was significant $(\mathrm{F}=22.5, \mathrm{df}=3 / 36, \mathrm{p}<.01)$. Duncan's multiple range test showed that the difference between the DRF and the FR-5 condition was significant, with the higher rate in the latter, but that the Ss which were to be extinguished after a 1 day delay did not differ reliably from those to be extinguished after a 21 day delay.

\section{Extinction}

An analysis of variance of the percentage of free feeding body weight on the day of extinction revealed no significant differences between groups.

Extinction measures were rate of responding in the first $35 \mathrm{~min}$. of extinction and the total number of responses to a criterion of $10 \mathrm{~min}$. without responding. When plotted, both measures showed quite similar relationships. However, the great variability in time to the $10 \mathrm{~min}$. extinction criterion prompted concentration on the initial rate measure in the remainder of the analysis.

The mean rates of responding for the four experimental conditions in the first $35 \mathrm{~min}$. of extinction are shown in Fig. 1. An analysis of variance indicated that the main effect of reinforcement schedule was significant

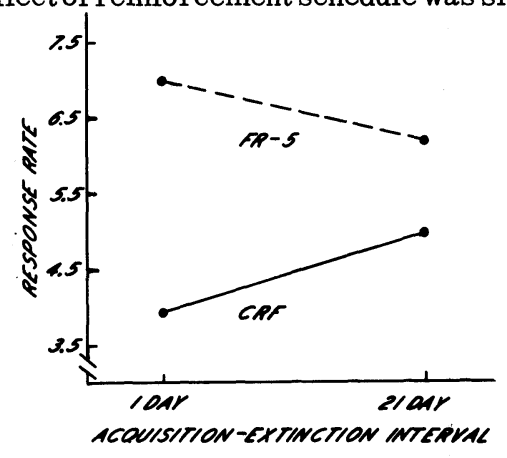

Fig. 1. Mean responses $/ \mathrm{min}$. in the first $35 \mathrm{~min}$. of extinction. Note: each point is based on data from a separate group.

$(\mathrm{F}=23.7, \mathrm{df}=1 / 36, \mathrm{p}<.01)$, that the delay interval was not significant, but that the interaction between the two variables was significant $(F=4.40, \mathrm{df}=1 / 36, \mathrm{p}<.05)$. Tests of simple main effects of reinforcement schedule were significant for the 1 day delay condition $(F=24.0$, $\mathrm{df}=1 / 36, \mathrm{p}<.01$ ), but not for the 21 day delay condition. Examination of Fig. 1 indicates that the higher CRF rate combined with the lower FR-5 rate following the 21 day delay accounts for the significant interaction.

\section{Discussion}

In an S-paced response situation, such as the bar press presents, an intermittent schedule of reinforcement finally results in higher response rates during acquisition, due in some degree simply to the differing times required to consume the reinforcement. This higher rate is evidenced in the data presented, and may be responsible in part at least for the higher response rate of the FR-5 Ss during extinction in the 1 day delay condition. However, by the end of three weeks, the data show that extinction response rates are not reliably different, due both to the higher response rates of the Ss under the CRF contingency and the lower rate under the FR-5 contingency. It might be argued that this is confirmatory of a discrimination hypothesis of the partial reinforcement effect. That is, over the three week period the animals might have forgotten the reinforcement contingencies they were under, so that discrimination of the onset of extinction was more nearly the same for the groups than after only $24 \mathrm{hr}$. "Forgetting of contingencies" is reminiscent of Gleitman \& Bernheim's (1963) description of their data on fixed interval reinforcement forgetting as attributable not to a failure to retain the motor response, but rather to a forgetting of when not to respond. It is also related to Perkins \& Weyant's (1958) interpretation of their data on the failure of generalization decrement after a delay as a forgetting of the stimulus properties of the environment. In the context of the present data, a possible specification of what is meant by forgetting of contingencies can be obtained by utilizing what is known about the effect of a delay interval on the slope of certain stimulus generalization functions (Thomas \& Lopez, 1962). There it is shown that delay brings about a flattening of the gradient of generalization of a discriminative stimulus (wavelength of light) controlling key pecking in the pigeon, without decreasing the absolute response rate, implying that the tendency to respond to generalized stimuli is up somewhat following a delay.

Applying this to the data at hand, let S-1 stand for the sound intensity associated with the pellet dispenser, relays, and counter when a bar press results in reinforcement, and S-2 for the less intense sound associated with bar presses which do not result in reinforcement. In acquisition, the CRF groups are exposed only to $\mathrm{S}-1$, which comes to control food cup approaches and eating; stimuli from which in turn control further bar presses. During extinction, S-2 is a generalized stimulus which, if the generalization slope data are relevant, controls a stronger food cup $\longrightarrow$ bar press sequence after a three week delay than after $24 \mathrm{hr}$. On the other hand, the FR-5 groups learn two response chains during acquisition. They are, $\mathrm{S}-1 \longrightarrow$ food cup $\rightarrow$ bar press, and $\mathrm{S}-2 \longrightarrow$ bar press. Flattening of the generalization gradients in this case would result in a greater overlap of the stimulus gradients controlling the two chains. One result of this would be that during extinction, S-2 would more often control food cup approaches after three weeks than after $24 \mathrm{hr}$., with a resulting decline in bar-pressing rate.

\section{References}

Gleitman, H., \& Bernheim, J. W. Retention of fixed-interval performance in rats. J. comp. physiol. Psychol., 1963, 56, 839-841.

Gleitman, H., Steinman, Fredda, \& Bernheim, J. W. Effect of prior interference upon retention of fixed-interval performance in rats. J. comp. physiol. Psychol., 1965, 59, 461-462.

Perkins, C. C., Jr., \& Weyant, B. G. The interval between training and test trials as a determiner of the slope of generalization gradients. J. comp. physiol. Psychol., 1958, 51, 596-600.

Thomas, D. R., \& Lopez, L. J. The effects of delayed testing on generalization slope. J. comp. physiol. Psychol., 1962, 55, 541-544.

Note

1. This research was supported, in part, by National Science Foundation Grant G-6161. 